\title{
Stability of Perturbed Set Differential Equations Involving Causal Operators in Regard to Their Unperturbed Ones considering Difference in Initial Conditions
}

\author{
Coșkun Yakar (D) and Hazm Talab \\ Department of Mathematics, Faculty of Science, Gebze Technical University, Gebze, 141-41400 Kocaeli, Turkey \\ Correspondence should be addressed to Hazm Talab; h.talab@gtu.edu.tr
}

Received 23 June 2021; Accepted 21 July 2021; Published 2 August 2021

Academic Editor: Maria L. Gandarias

Copyright (C) 2021 Coșkun Yakar and Hazm Talab. This is an open access article distributed under the Creative Commons Attribution License, which permits unrestricted use, distribution, and reproduction in any medium, provided the original work is properly cited.

\begin{abstract}
We investigate the stability of solutions of perturbed set differential equations with causal operators in regard to their corresponding unperturbed ones considering the difference in initial conditions (time and position) by utilizing Lyapunov functions and Lyapunov functionals.
\end{abstract}

\section{Introduction}

Set differential equations (SDEs) have received a lot of attention in recent decades, emerging as an independent discipline. The unifying approach of the SDEs [1-4] is one of their significant points, which is also considered as an advantage. It has been observed that SDEs are generalized forms of vector and nonlinear ordinary differential equations (ODEs) [5-7] and that ODEs can be considered as special cases of SDEs while studying its stability properties in a semilinear metric space. Moreover, SDEs can play an important role in examining multivalued differential equations and inclusions [2] and also in fuzzy equations [8]. Furthermore, causal operators [9-11] encompass a wide range of ODEs and integral [12] and integro-differential equations [13]. Thus, SDEs involving causal operators [14] give a comprehensive form of diverse classes of equations as they embrace the aforementioned special cases of differential equations.

On other hand, stability analysis $[12,14-21]$ can be useful for determining the qualitative properties, which in its turn leads to a more perceptive behavioral look of the differential equation's solutions, even when they are unknown explicitly $[5,6]$.

In real-life problems, the solutions of differential equations may differ in initial conditions (time or position). To deal with such cases, initial time difference (ITD) stability analysis [15-17, 22-27] compares the behavioral properties of the solutions considering the change in initial conditions [22, 24-27].

Such generalized approach of stability plays an important role in the qualitative theory of differential equations in analysing the stability and other behavioral properties of its solutions using a more realistic manner that considers the difference in time and position in the initial state.

Diverse forms of ITD stability have been investigated in analysing the solutions for many forms of differential equations (such as, ordinary, fractional, and fuzzy).

In this work, we study ITD stability results for SDEs involving causal operators, by employing Lyapunov functions and functionals. We give sufficient conditions to ITD stability and ITD asymptotic stability.

We laid the foundations in Section 2, by investigating the basic definitions and results regarding ITD stability and stability of null solution of SDE with causal operator. In Section 3 , we present the difficulties we face when we try to infer ITD stability properties from those of null solution, by comparing both classical and ITD notions of stability of the solution of the SDE with causal operator. In Section 4, we establish the comparison theorems for initial time difference that resolve the complications regarding the classical notion stability. In 
Section 5, we present an approach that resolve the difficulties allowing us to infer stability properties for solution of the perturbed form of SDE involving causal operator corresponding to the unperturbed one, by using a suitable comparison system.

\section{Preliminaries}

Let $K\left(\mathbb{R}^{n}\right)$ denotes all compact nonempty subsets of $\mathbb{R}^{n}$ and $K_{c}\left(\mathbb{R}^{n}\right)$ denotes all compact and convex nonempty subsets of $\mathbb{R}^{n}$. The Hausdorff metric between any bounded sets $A_{1}$ and $A_{2}$ in $\mathbb{R}^{n}$ is defined by

$$
D\left(A_{1}, A_{2}\right)=\max \left[\sup _{a_{2} \in A_{2}} d\left(a_{2}, A_{1}\right), \sup _{a_{1} \in A_{1}} d\left(a_{1}, A_{2}\right)\right],
$$

where

$$
d\left(a_{2}, A_{1}\right)=\inf \left\{d\left(a_{2}, a_{1}\right): a_{1} \in A_{1}\right\} .
$$

Each of $\left(K\left(\mathbb{R}^{n}\right), D\right)$ and $\left(K_{c}\left(\mathbb{R}^{n}\right), D\right)$ forms a complete metric space. $K_{c}\left(\mathbb{R}^{n}\right)$ with ordinary addition and nonnegative scalar multiplication is a semilinear metric space that can be regarded as a cone in an appropriate Banach space.

Some properties of $D$ can be stated as follows:

$$
\begin{aligned}
& \text { (1) } D\left(A_{1}, A_{2}\right)=D\left(A_{2}, A_{1}\right), \\
& \text { (2) } D\left(A_{1}+C, A_{2}+C\right)=D\left(A_{1}, A_{2}\right), \\
& \text { (3) } D\left(k A_{1}, k A_{2}\right)=k D\left(A_{1}, A_{2}\right), \\
& \text { (4) } D\left(A_{1}, A_{2}\right) \leq D\left(A_{1}, C\right)+D\left(C, A_{2}\right) \text {, }
\end{aligned}
$$

for any $A_{1}, A_{2}, C \in K_{c}\left(\mathbb{R}^{n}\right)$ and $k \in \mathbb{R}_{+}$, where $A_{1}+C$ denotes $\left\{a_{1}+c: a_{1} \in A_{1}, c \in C\right\}$ and the scalar multiplication $k A_{1}$ denotes $\left\{k a_{1}: a_{1} \in A_{1}\right\}$. If $k=-1$, we get $-A_{1}=$ $(-1) A_{1}=\left\{-a_{1}: a_{1} \in A_{1}\right\}$.

In general, $A_{1}+\left(-A_{1}\right) \neq\{0\}$ (unless $A_{1}=\{a\}$ is a singleton). To overcome with this implication of Minkowski difference, i.e.,

$$
A_{1}+(-1) A_{2}=\left\{a_{1}-a_{2}: a_{1} \in A_{1}, a_{2} \in A_{2}\right\} .
$$

Hukuhara difference between $A_{1}, A_{2} \in K_{c}\left(\mathbb{R}^{n}\right)$ is introduced as follows:

If there is a $C \in K_{c}\left(\mathbb{R}^{n}\right)$ where $C+A_{2}=A_{1}$, then Hukuhara difference exists and it is denoted by $A_{1} \ominus A_{2}$, or simply $A_{1}-A_{2}$ when there is no confusion with Minkowski difference, i.e., $A_{1} \ominus A_{2}=C \Longleftrightarrow C+A_{2}=A_{1}$.

Hukuhara difference has an important property, Minkowski difference does not have (in general), which is $A_{1}-$ $A_{1}=\{0\}$, for any compact and convex nonempty subset $A_{1}$ of $\mathbb{R}^{n}$.

Let $U: I \longrightarrow K_{c}\left(\mathbb{R}^{n}\right)$ be a given multifunction, where $I$ denotes a real-number interval. $U$ is said to be Hukuhara differentiable at $t_{0} \in I$, if we ensure the existence of $D_{H} U\left(t_{0}\right) \epsilon$ $K_{c}\left(\mathbb{R}^{n}\right)$ so

$$
\lim _{\varepsilon \longrightarrow 0^{+}} \frac{U\left(t_{0}+\varepsilon\right)-U\left(t_{0}\right)}{\varepsilon} \text { and } \lim _{\varepsilon \longrightarrow 0^{+}} \frac{U\left(t_{0}\right)-U\left(t_{0}-\varepsilon\right)}{\varepsilon},
$$

both exist in $K_{c}\left(\mathbb{R}^{n}\right)$ and have the same value as $D_{H} U\left(t_{0}\right)$.

The existence of $U\left(t_{0}+\varepsilon\right)-U\left(t_{0}\right)$ and $U\left(t_{0}\right)-U\left(t_{0}-\varepsilon\right)$, for a sufficiently small $\varepsilon>0$, is implicit in $D_{H} U\left(t_{0}\right)$ definition.

By considering $K_{c}\left(\mathbb{R}^{n}\right)$ as a complete cone, and embedding it in an appropriate Banach space, with the properties of Bochner integral, it is seen that if

$$
G(t)=G\left(t_{0}\right)+\int_{t_{0}}^{t} F(s) d s, \quad t \in I,
$$

where $F: I \longrightarrow K_{c}\left(\mathbb{R}^{n}\right)$ is integrable in Bochner sense, then $G$ is Hukuhara differentiable, i.e., $D_{H} G(t)$ exits, and $D_{H} G(t)$ $=F(t)$ holds almost everywhere on $I$.

The Hukuhara integral of $F$, over a compact set $I \subset \mathbb{R}_{+}$, is defined as the integral of a continuous selector $f$ of $F$ over $I$.

$$
\int_{I} F(t) d t=\left[\int_{I} f(t) d t\right] .
$$

Considering the null element $\theta$ of $\mathbb{R}^{n}$ as a point set, let us define $\|$.$\| on the space E=C\left[\left[t_{0}, \infty\right), K_{c}\left(\mathbb{R}^{n}\right)\right]$ as

$$
\|U\|=\sup _{s \in\left[t_{0}, \infty\right)} \frac{D[U(s), \theta]}{h(s)}<\infty
$$

where $U \in E$ and $h:\left[t_{0}, \infty\right) \longrightarrow \mathbb{R}_{+}$is a continuous map. Then, $(E,\|\|$.$) is a complete normed space.$

The operator $Q \in C[E, E]$ is called causal if $U_{1}(s)=U_{2}(s)$, for $U_{1}, U_{2} \in E$, and $s \in\left[t_{0}, t\right]$ implies

$$
\left(Q U_{1}\right)(s)=\left(Q U_{2}\right)(s), \quad s \in\left[t_{0}, t\right] .
$$

Consider the following equations:

$$
\begin{aligned}
& \begin{array}{l}
D_{H} U=(Q U)(t), U\left(t_{0}\right)=U_{0}, \\
\text { for } U_{0} \in K_{c}\left(\mathbb{R}^{n}\right) \text { and } t \geq t_{0} \geq 0, \\
D_{H} U=(Q U)(t), U\left(\tau_{0}\right)=V_{0}, \\
\text { for } V_{0} \in K_{c}\left(\mathbb{R}^{n}\right) \text { and } t \geq \tau_{0} \geq 0, \\
D_{H} V=(P V)(t), V\left(\tau_{0}\right)=V_{0}, \\
\quad \text { for } V_{0} \in K_{c}\left(\mathbb{R}^{n}\right) \text { and } t \geq \tau_{0}, \\
D_{H} W=(S W)(t), W\left(\tau_{0}\right)=V_{0}-U_{0}, \\
\text { for } W\left(\tau_{0}\right)=W_{0} \in K_{c}\left(\mathbb{R}^{n}\right) \text { and } t \geq \tau_{0},
\end{array}
\end{aligned}
$$

where (10) and (11) are different in initial time and position, (12) is the perturbed form corresponding to the unperturbed system (11), and where $Q, P, S: E \longrightarrow E$ are causal operators and $S_{\rho}=\left\{U \in K_{c}\left(\mathbb{R}^{n}\right): D[U, \tilde{0}]<\rho<\infty\right\}$. 
A special case of (12) is

$$
(P V)(t)=(Q V)(t)+(R V)(t)
$$

where $(R V)(t)$ is the perturbation term.

Assume that $(Q \tilde{0})(t) \equiv \tilde{0}$ for $t \geq 0$, and assume the necessary smoothness of $P, Q$, and $R$ to guarantee the existence and uniqueness of the solution $U(t)=U\left(t, t_{0}, U_{0}\right)$ of (10) through $\left(t_{0}, U_{0}\right)$ for $t \geq t_{0}$ and those of the solution $V(t)=$ $V\left(t, \tau_{0}, V_{0}\right)$ of (12) through $\left(\tau_{0}, V_{0}\right)$ for $t \geq \tau_{0}$, in addition to their continuous dependence on initial conditions.

If $U \in C^{1}\left[J_{1}, K_{c}\left(\mathbb{R}^{n}\right)\right]$ on $J_{1}=\left[t_{0}, t_{0}+T_{1}\right]$, then it is said to be a solution of (10) on $J_{1}$ if (10) holds with this $U$ for all $t \in J_{1}$. If $U, V$, and $W \in C^{1}\left[J_{2}, K_{c}\left(\mathbb{R}^{n}\right)\right]$ on $J_{2}=\left[t_{0}, t_{0}+\right.$ $\left.T_{2}\right]$, then these are said to be solutions of (11), (12), and (13) on $J_{2}$ provided that they satisfy (11), (12), and (13) on $J_{2}$, respectively.

The continuously differentiability of $U(t), V(t)$ and $W(t)$ enables us to write

$$
\begin{array}{cc}
U(t)=U_{0}+\int_{t_{0}}^{t} D_{H} U(s) d s, & U_{0} \in K_{c}\left(\mathbb{R}^{n}\right) \text { and } t \in J_{1}, \\
U(t)=U_{0}+\int_{\tau_{0}}^{t} D_{H} U(s) d s, & U_{0} \in K_{c}\left(\mathbb{R}^{n}\right) \text { and } t \in J_{2}, \\
V(t)=V_{0}+\int_{\tau_{0}}^{t} D_{H} V(s) d s, & V_{0} \in K_{c}\left(\mathbb{R}^{n}\right) \text { and } t \in J_{2}, \\
W(t)=W_{0}+\int_{\tau_{0}}^{t} D_{H} W(s) d s, & W_{0} \in K_{c}\left(\mathbb{R}^{n}\right) \text { and } t \in J_{2} .
\end{array}
$$

Thus, the corresponding equations with the initial value problems (IVPs) of (10), (11), (12), and (13) are the followings, respectively.

$$
\begin{aligned}
& U(t)=U_{0}+\int_{t_{0}}^{t}(Q U(s))(s) d s, \quad U_{0} \in K_{c}\left(\mathbb{R}^{n}\right) \text { and } t \in J_{1}, \\
& U(t)=U_{0}+\int_{\tau_{0}}^{t}(Q U(s))(s) d s, \quad U_{0} \in K_{c}\left(\mathbb{R}^{n}\right) \text { and } t \in J_{2}, \\
& V(t)=V_{0}+\int_{\tau_{0}}^{t}(P V(s))(s) d s, \quad V_{0} \in K_{c}\left(\mathbb{R}^{n}\right) \text { and } t \in J_{2}, \\
& W(t)=W_{0}+\int_{\tau_{0}}^{t}(S W(s))(s) d s, \quad W_{0} \in K_{c}\left(\mathbb{R}^{n}\right) \text { and } t \in J_{2},
\end{aligned}
$$

where the integrals are the Hukuhara integrals.

Note that $U(t)$ and also $U(t), V(t)$, and $W(t)$ are solutions of (10) and (11), (12), and (13) if and only if they satisfy (16) on $J_{1}$ and (17), (18), and (19) on $J_{2}$, respectively.
Furthermore, to resemble the behavioral properties of solution of (10) with these of solution of an ordinary correspondent one, we assume that $U_{0}=Z_{0}+U_{0}^{*}$ to ensure the existence of the Hukuhara difference $U_{0}^{*}=U_{0}-Z_{0}$. Accordingly, we have the solution $U\left(t, t_{0}, U_{0}-Z_{0}\right)=U\left(t, t_{0}, U_{0}^{*}\right)$ and the corresponding equation

$$
\begin{aligned}
& D_{H} U=(Q U)(t), U\left(t_{0}\right)=U_{0}^{*}, \\
& \qquad \text { for } U_{0}^{*} \in K_{c}\left(\mathbb{R}^{n}\right) \text { and } t \geq t_{0} \geq 0 .
\end{aligned}
$$

We also assume that $V_{0}=S_{0}+V_{0}^{*}$ so that the Hukuhara difference $V_{0}^{*}=V_{0}-S_{0}$ exists. Consequently, we have the solution $V\left(t, t_{0}, V_{0}-S_{0}\right)=U\left(t, t_{0}, V_{0}^{*}\right)$ and the corresponding equation

$$
\begin{aligned}
& D_{H} U=(Q U)(t), U\left(\tau_{0}\right)=V_{0}^{*}, \\
& \text { for } V_{0}^{*} \in K_{c}\left(\mathbb{R}^{n}\right) \text { and } t \geq \tau_{0} \geq 0,
\end{aligned}
$$

and the corresponding perturbed system of (21)

$$
\begin{aligned}
& D_{H} V=(P V)(t), V\left(\tau_{0}\right)=V_{0}^{*}, \\
& \qquad \text { for } V_{0}^{*} \in K_{c}\left(\mathbb{R}^{n}\right) \text { and } t \geq \tau_{0} \geq 0 .
\end{aligned}
$$

If we ensure the existence of aforementioned Hukuhara differences and there is no ambiguity in the context, we may omit the asterisk symbols in (20), (21), and (22) and continue with the familiar notation as in (10), (11), and (12).

Before establishing the comparison theorems and criteria for ITD stability for SDEs involving causal operators, let us present the following basic definitions for ITD stability.

Definition 1. Let $\tilde{U}\left(t, \tau_{0}, U_{0}\right)=U\left(t-\eta, t_{0}, U_{0}\right)$, where $U(t$, $\left.t_{0}, U_{0}\right)$ solves (10) for $t \geq \tau_{0}, \tau_{0}=\eta+t_{0}$ and $t_{0}, \tau_{0} \in \mathbb{R}_{+}$. The solution $V\left(t, \tau_{0}, V_{0}\right)$ of (12) for $t \geq \tau_{0}, \tau_{0} \in \mathbb{R}_{+}$with the initial conditions $\left(\tau_{0}, V_{0}\right)$ is called

(S1) An ITD stable solution w.r.t. the solution $\tilde{U}\left(t, \tau_{0}\right.$, $\left.U_{0}\right)$ if and only if for any given $\varepsilon>0$, we can designate two positive values $\delta_{1}=\delta_{1}\left(\varepsilon, \tau_{0}\right)$ and $\delta_{2}=\left(\varepsilon, \tau_{0}\right)$ which give us the following inequality:

$$
D\left[V\left(t, \tau_{0}, V_{0}\right)-U\left(t-\eta, t_{0}, U_{0}\right), \tilde{0}\right]<\varepsilon
$$

whenever $D\left[V_{0}-U_{0}, \tilde{0}\right]<\delta_{1}$ and $d_{s}\left[\tau_{0}-t_{0}, 0\right]<\delta_{2}$ for $t \geq \tau_{0}$.

(S2) An ITD uniformly stable solution w.r.t. the solution $\tilde{U}\left(t, \tau_{0}, U_{0}\right)$ if both $\delta_{1}, \delta_{2}$ in (S1) are independent of $\tau_{0} \in \mathbb{R}$

(S3) An ITD attractive solution w.r.t. the solution $\tilde{U}(t$, $\left.\tau_{0}, U_{0}\right)$ if and only if for any $\varepsilon, \tau_{0}>0$, we can designate two positive values $\delta_{1}=\delta_{1}\left(\tau_{0}\right), \delta_{2}=\delta_{2}\left(\tau_{0}\right)$ and a $T=T\left(\varepsilon, \tau_{0}\right)$ which give us the following inequality:

$$
D\left[V\left(t, \tau_{0}, V_{0}\right)-U\left(t-\eta, t_{0}, U_{0}\right), \tilde{0}\right]<\varepsilon
$$

provided that $D\left[V_{0}-U_{0}, \tilde{0}\right]<\delta_{1}$ and $d_{s}\left[\tau_{0}-t_{0}, 0\right]<\delta_{2}$ for $t$ $\geq \tau_{0}+T$ 
(S4) An ITD uniformly attractive solution w.r.t. the solution $\tilde{U}\left(t, \tau_{0}, U_{0}\right)$ if and only if all $\delta_{1}>0, \delta_{2}>0$, and $T>0$ in (S3) are independent of $\tau_{0} \in \mathbb{R}_{+}$

(S5) An ITD asymptotically stable solution w.r.t. the solution $\tilde{U}\left(t, \tau_{0}, U_{0}\right)$ if and only if (S1) and (S3) hold all together, or equivalently if (S1) holds and there exist $\gamma_{1}\left(\tau_{0}\right)$ $>0$ and $\gamma_{2}\left(\tau_{0}\right)>0$ so that

$$
\lim _{t \longrightarrow \infty} D\left[V\left(t, \tau_{0}, V_{0}\right)-U\left(t-\eta, t_{0}, U_{0}\right), \tilde{0}\right]=0,
$$

for any $V\left(t, \tau_{0}, V_{0}\right)$ and $U\left(t-\eta, t_{0}, U_{0}\right)$ with

$$
D\left[V_{0}-U_{0}, \tilde{0}\right]<\gamma_{1} \text {, and } d_{s}\left[\tau_{0}-t_{0}, 0\right]<\gamma_{2}, \quad \text { for } t \geq \tau_{0} \text {. }
$$

(S6) An ITD uniformly asymptotically stable solution w.r.t. the solution $\tilde{U}\left(t, \tau_{0}, U_{0}\right)$ if and only if (S2) and (S4) hold all together, or equivalently if in addition to (S2) we can designate, for any given $\varepsilon>0$, two positive values $\gamma_{1}=$ $\gamma_{1}(\varepsilon)$ and $\gamma_{2}=\gamma_{2}(\varepsilon)$ and a $T(\varepsilon)>0$ so

$$
D\left[V\left(t, \tau_{0}, V_{0}\right)-U\left(t-\eta, t_{0}, U_{0}\right), \tilde{0}\right]<\varepsilon,
$$

provided that $D\left[V_{0}-U_{0}, \tilde{0}\right]<\gamma_{1}$ and $d_{s}\left[\tau_{0}-t_{0}, 0\right]<\gamma_{2}$ for each $t \geq \tau_{0}+T(\varepsilon)$; or if $\gamma_{1}$ and $\gamma_{2}$ in (S5) are independent of $\tau_{0} \in \mathbb{R}_{+}$

(S7) An ITD exponentially asymptotically stable solution w.r.t. the solution $\tilde{U}\left(t, \tau_{0}, U_{0}\right)$ if and only if there exists a constant $\alpha>0$ such that

$$
\begin{aligned}
& D\left[V\left(t, \tau_{0}, V_{0}\right)-U\left(t-\eta, t_{0}, U_{0}\right), \tilde{0}\right] \\
& \quad \leq D\left[V_{0}-U_{0}, \tilde{0}\right] \exp \left[-\alpha\left(t-\tau_{0}\right)\right]
\end{aligned}
$$

for $t \geq \tau_{0} \in \mathbb{R}_{+}$.

Definition 2. The function $\sigma \in C\left[[0, \rho), \mathbb{R}_{+}\right]$is said to be from the class $\mathbb{K}$, or simply written as $\sigma \in \mathbb{K}$, if and only if it associates zero to zero and $\sigma(\lambda)$ is strictly increasing in $\lambda$. If $\rho$ $=\infty$ and $\sigma(\lambda) \longrightarrow \infty$ as $\lambda \longrightarrow \infty$, then it is said to be from the class $\mathbb{K}_{\infty}$, or simply written as $\sigma \in \mathbb{K}_{\infty}$.

Definition 3. Let $L \in C\left[\mathbb{R}_{+} \times K_{c}\left(\mathbb{R}^{n}\right), \mathbb{R}_{+}\right]$,

(a) The Dini derivatives of $L$ are defined as

$$
\begin{aligned}
& D^{+} L(t, V)=\lim _{\varepsilon \longrightarrow 0^{+}} \sup \frac{1}{\varepsilon}[L(t+\varepsilon, V+\varepsilon(Q V)(t))-L(t, V)], \\
& D_{-} L(t, V)=\lim _{\varepsilon \longrightarrow 0^{-}} \inf \frac{1}{\varepsilon}[L(t+\varepsilon, V+\varepsilon(Q V)(t))-L(t, V)],
\end{aligned}
$$

for $(t, V) \in \mathbb{R}_{+} \times K_{c}\left(\mathbb{R}^{n}\right)$. (b) The generalized (Dini-like) derivatives of $L$ are defined as

$$
\begin{aligned}
D_{*}^{+} L( & t, V-\tilde{U}) \\
= & \lim _{\varepsilon \longrightarrow 0^{+}} \sup \frac{1}{\varepsilon}[L(t+\varepsilon, V-\tilde{U} \\
& +\varepsilon((P V)(t)-(\tilde{Q} \tilde{U})(t)))-L(t, V-\tilde{U})], \\
D_{*-} L(t, V-\tilde{U}) & \\
= & \lim _{\varepsilon \longrightarrow 0^{-}} \inf \frac{1}{\varepsilon}[L(t+\varepsilon, V-\tilde{U} \\
& +\varepsilon((P V)(t)-(\tilde{Q} \tilde{U})(t)))-L(t, V-\tilde{U})],
\end{aligned}
$$

where $V\left(t, \tau_{0}, V_{0}\right)$ solves (12) and $\tilde{U}\left(t, \tau_{0}, U_{0}\right)=$ $U\left(t-\eta, t_{0}, U_{0}\right)$ and $U\left(t, t_{0}, U_{0}\right)$ solve (10) for $t \geq$ $\tau_{0}, t_{0}, \tau_{0} \in \mathbb{R}_{+}$, where $\tau_{0}=\eta+t_{0}$.

(c) The generalized derivatives of a Lyapunov functional $L(t, W) \in C\left[\mathbb{R}_{+} \times E, \mathbb{R}_{+}\right]$are defined as

$$
\begin{aligned}
D^{+} L & (t, V(t)-\tilde{U}(t)) \\
= & \lim _{\varepsilon \longrightarrow 0^{+}} \sup \frac{1}{\varepsilon}[L(t+\varepsilon, V(t+\varepsilon, t, V) \\
& -\tilde{U}(t+\varepsilon, t, \tilde{U}))-L(t, V(t)-\tilde{U}(t))], \\
D_{-} L & (t, V(t)-\tilde{U}(t)) \\
= & \lim _{\varepsilon \longrightarrow 0^{-}} \inf \frac{1}{\varepsilon}[L(t+\varepsilon, V(t+\varepsilon, t, V) \\
& -\tilde{U}(t+\varepsilon, t, \tilde{U}))-L(t, V(t)-\tilde{U}(t))],
\end{aligned}
$$

where $V(t+\varepsilon, t, V)$ solves (12) and $\tilde{U}(t+\varepsilon, t, \tilde{U})$ solves (10). Furthermore, $\tilde{U}(t)=U(t-\eta)$, where $U(t)=U\left(t, t_{0}, U_{0}\right)$ solves (10) for $t \geq \tau_{0}$.

In the following section, let us present a comparative analysis on how we can infer the stability properties from those of the null solution of the SDE with causal operator in the classical sense of stability, whereas there is incompatibility in using the same manner in the context of ITD stability.

\section{Classical vs. ITD Stability of SDE with Causal Operator}

3.1. Classical Notion of Stability of SDE with Causal Operator. Consider the following SDE with causal operator

$$
\begin{aligned}
& D_{H} V=(Q V)(t), V\left(\tau_{0}\right)=V_{0}, \\
& \quad \text { for } V_{0} \in K_{c}\left(\mathbb{R}^{n}\right) \text { and } t \geq \tau_{0}, \tau_{0} \in \mathbb{R}_{+},
\end{aligned}
$$

where $Q: E \longrightarrow E$ and $S_{\rho}=\left\{V \in K_{c}\left(\mathbb{R}^{n}\right): D[V, \tilde{0}]<\rho<\infty\right\}$.

Let $V\left(t, t_{0}, V_{0}\right)$ be any solution of (32) and assume that $(Q \tilde{0})(t)=\tilde{0}, t \in \mathbb{R}_{+}$so that the null solution $V \equiv 0$ solves (32) with the initial conditions $\left(t_{0}, 0\right)$. 
Definition 4. The null solution $V=0$ of (32) is called

(S1) A stable solution if and only if given any $\varepsilon>0, \tau_{0}$ $\in \mathbb{R}_{+}$, we can designate two positive functions $\delta_{1}=\delta_{1}\left(\varepsilon, \tau_{0}\right)$ and $\delta_{2}=\delta_{2}\left(\varepsilon, \tau_{0}\right)$, which are continuous in $\tau_{0}$ for each $\varepsilon$, and which give us

$$
D\left[V\left(t, \tau_{0}, V_{0}\right), \tilde{0}\right]<\varepsilon,
$$

for $t \geq \tau_{0}$, given that $D\left[V_{0}, \tilde{0}\right]<\delta_{1}$ and $d_{s}\left[\tau_{0}, 0\right]<0$.

(S2) A uniformly stable solution if additionally $\delta_{1}$ and $\delta_{2}$ are independent of $\tau_{0}$

(S3) A quasiequiasymptotically stable solution if and only if given any positive $\varepsilon, t_{0}$ we can designate $\delta_{0}=\delta_{0}\left(t_{0}\right)$ and $T=T\left(t_{0}, \varepsilon\right)$ so

$$
D\left[V\left(t, \tau_{0}, V_{0}\right), \tilde{0}\right]<\varepsilon,
$$

for $t \geq t_{0}+T$ provided that $D\left[V_{0}, \tilde{0}\right]<\delta_{0}$.

(S4) A quasiuniformly asymptotically stable solution if additionally each of $\delta_{0}$ and $T$ in (S3) is independent of $t_{0}$

(S5) An equiasymptotically stable solution if and only if both (S1) and (S3) are verified

(S6) A uniformly asymptotically stable solution if and only if both (S2) and (S4) are verified

(S7) A quasiequiasymptotically stable solution if and only if given any positive values $\varepsilon, \alpha, t_{0}$, we can designate $T=$ $T\left(t_{0}, \varepsilon, \alpha\right)>0$ which gives us

$$
D\left[V\left(t, \tau_{0}, V_{0}\right), \theta\right]<\varepsilon,
$$

for $t \geq t_{0}+T$, provided that $D\left[V_{0}, \tilde{0}\right] \leq \alpha$.

(S8) A quasiuniformly asymptotically stable solution if and only if (S7) is verified with $T$ being independent of $t_{0}$ additionally

(S9) A completely stable solution if and only if (S1) holds and (S7) is verified given any $\alpha, 0 \leq \alpha<\infty$

(S10) A uniformly completely stable solution if and only if (S2) holds and (S8) is verified for all $\alpha, 0 \leq \alpha<\infty$

(S11) an unstable solution if and only if (S1) fails to hold.

Definition 5. The solution $V\left(t, \tau_{0}, V_{0}\right)$ of (32) with the initial conditions $\left(\tau_{0}, V_{0}\right)$ is called a stable solution w.r.t. the solution $V\left(t, \tau_{0}, U_{0}\right)$ of (32) for $t \geq t_{0} \geq 0$ if and only if for each $\varepsilon>0$, we can designate $\delta_{1}=\delta_{1}\left(\varepsilon, \tau_{0}\right)$ and $\delta_{2}=\delta_{2}\left(\varepsilon, \tau_{0}\right)$ which are positive and continuous in $\tau_{0}$ for each $\varepsilon$ so

$$
D\left[V\left(t, \tau_{0}, V_{0}\right)-V\left(t, \tau_{0}, U_{0}\right), \tilde{0}\right]<\varepsilon,
$$

provided that $D\left[V_{0}-U_{0}, \tilde{0}\right]<\delta_{1}$ and $d_{s}\left[\tau_{0}, 0\right]<\delta_{2}$ for $t \geq \tau_{0}$.

Additionally, if each of $\delta_{1}$ and $\delta_{2}$ is independent of $\tau_{0} \epsilon$ $\mathbb{R}_{+}$, then the solution $V\left(t, \tau_{0}, V_{0}\right)$ of (32) is called a uniformly stable solution w.r.t. the solution $V\left(t, \tau_{0}, U_{0}\right)$.

Remark 6. Let $V\left(t, t_{0}, V_{0}\right)$ be a given solution of (32). We can use the following change of variable to study the stability of this solution.
For $t \geq t_{0}$, set

$$
\Omega\left(t, t_{0}, V_{0}-U_{0}\right)=V\left(t, t_{0}, V_{0}\right)-V\left(t, t_{0}, U_{0}\right)
$$

Then,

$$
D_{H} \Omega\left(t, t_{0}, V_{0}-U_{0}\right)=D_{H} V\left(t, t_{0}, V_{0}\right)-D_{H} V\left(t, t_{0}, U_{0}\right),
$$

$$
\begin{aligned}
D_{H} \Omega( & \left(t, t_{0}, V_{0}-U_{0}\right) \\
= & \left(Q \Omega\left(t, t_{0}, V_{0}-U_{0}\right)\right)(t) \\
& +\left(Q V\left(t, t_{0}, U_{0}\right)\right)(t)-\left(Q V\left(t, t_{0}, U_{0}\right)\right)(t) \\
= & \left(Q\left(\Omega\left(t, t_{0}, V_{0}-U_{0}\right)+V\left(t, t_{0}, U_{0}\right)\right)\right) \\
& \cdot(t)-\left(Q V\left(t, t_{0}, U_{0}\right)\right)(t) \\
= & \left(\tilde{Q} \Omega\left(t, t_{0}, V_{0}-U_{0}\right)\right)(t) .
\end{aligned}
$$

If $V_{0}-U_{0}=\tilde{0}$, we can ascertain that $\Omega\left(t, t_{0}, V_{0}-U_{0}\right) \equiv \tilde{0}$ solves the transformed SDE. Hence, it indicates that (Q0̃) $(t)=\tilde{0}$ for $t \geq t_{0} \geq 0$.

Since $D_{H} \Omega=\tilde{0}$ and $\Omega\left(t, t_{0}, \tilde{0}\right)=\tilde{0}$, the solution $V\left(t, t_{0}\right.$, $U_{0}$ ) of (32) corresponds to the identically null solution of $D_{H} \Omega=(\tilde{Q} \Omega)(t)$ where

$$
\begin{aligned}
(\tilde{Q} & \left.\Omega\left(t, t_{0}, V_{0}-U_{0}\right)\right)(t) \\
= & \left(Q\left(\Omega\left(t, t_{0}, V_{0}-U_{0}\right)+V\left(t, t_{0}, U_{0}\right)\right)\right) \\
& \cdot(t)-\left(Q V\left(t, t_{0}, U_{0}\right)\right)(t) .
\end{aligned}
$$

Hence, without loss of generality, it is enough to consider the stability of the null solution $V\left(t, t_{0}, U_{0}\right) \equiv \tilde{0}$ of (32). Unfortunately, such procedure is not feasible for ITD stability analysis.

3.2. ITD Stability of SDE with Causal Operator. Assume $U(t$, $\left.\tau_{0}, V_{0}\right)$ solves $(11)$ and let $\tilde{U}\left(t, \tau_{0}, U_{0}\right)=U\left(t-\eta, t_{0}, U_{0}\right)$ where $U\left(t, t_{0}, U_{0}\right)$ solves (10) for $t \geq \tau_{0} \geq 0$. Let us go through a similar procedure as in (39) by setting

$$
\begin{aligned}
\Omega\left(t, \tau_{0}, V_{0}-U_{0}\right)= & U\left(t, \tau_{0}, V_{0}\right) \\
& -U\left(t-\eta, t_{0}, U_{0}\right), \quad \text { for } t \geq \tau_{0} .
\end{aligned}
$$

Then,

$$
\begin{gathered}
D_{H} \Omega\left(t, \tau_{0}, V_{0}-U_{0}\right)=D_{H} U\left(t, \tau_{0}, V_{0}\right)-D_{H} U\left(t-\eta, t_{0}, U_{0}\right), \\
D_{H} \Omega\left(t, \tau_{0}, V_{0}-U_{0}\right)=\left(\tilde{Q} \Omega\left(t, \tau_{0}, V_{0}-U_{0}\right)\right)(\eta ; t) .
\end{gathered}
$$

Considering that even when $V_{0}=U_{0}, \Omega\left(t, \tau_{0}, \tilde{0}\right)$ is neither zero nor it solves the transformed differential system; also, $U$ $\left(t-\eta, t_{0}, U_{0}\right)$ is not the exactly null solution of $D_{H} \Omega=(\tilde{Q} \Omega)$ $(\eta ; t)$. Consequently, it is not an option to infer ITD stability properties using stability properties of the null solution. 
This motivates us to search for other different approaches in studying such behavioral properties.

\section{Comparison Theorems for ITD Stability of SDEs with Causal Operators}

Based on our previous analysis and other's studies $[5,7,10]$, due to the differences between the ITD notion of stability and the classical one, it is seen that the behavioral analysis of the zero solution cannot be utilized in ITD stability. In what follows, we present an approach that resolves those complications and enables us to infer the stability properties of the solution $V\left(t, \tau_{0}, V_{0}\right)$ of (12) w.r.t. $\tilde{U}(t)=U\left(t-\eta, t_{0}, U_{0}\right)$ where $U\left(t, t_{0}, U_{0}\right)$ solves (10).

\section{Theorem 7. Let}

(i) $L(t, W) \in C\left[\mathbb{R}_{+} \times K_{c}\left(\mathbb{R}^{n}\right), \mathbb{R}_{+}\right]$be locally Lipschitzian in $W$; i.e.,

$$
\left|L\left(t, W_{1}\right)-L\left(t, W_{2}\right)\right| \leq K D\left[W_{1}-W_{2}, \tilde{0}\right]
$$

(ii) For $(t, V),(t, \tilde{U}) \in \mathbb{R}_{+} \times K_{c}\left(\mathbb{R}^{n}\right)$ and $G \in C\left[\mathbb{R}_{+} \times\right.$ $\left.\mathbb{R}_{+}, \mathbb{R}_{+}\right]$, it is considered

$$
D_{*}^{+} L(t, V-\tilde{U}) \leq G(t, L(t, V(t)-\tilde{U}(t))), \quad t \geq \tau_{0},
$$

where

$U \in E_{1}=\left\{\Omega \in K_{c}\left(\mathbb{R}^{n}\right): L(s, \Omega(s)) \leq L(t, \Omega(t)), \quad t_{0} \leq s \leq t\right\}$,

$$
\begin{aligned}
& \text { and } \\
& \begin{aligned}
D_{*}^{+} L(t, V(t)-\tilde{U}(t)) \\
=\lim _{\varepsilon \longrightarrow 0^{+}} \sup \frac{1}{\varepsilon}[L(t+\varepsilon, V(t)-\tilde{U}(t) \\
\quad+\varepsilon((P V)(t)-(\tilde{Q} \tilde{U})(t)))-L(t, V(t)-\tilde{U}(t))] .
\end{aligned}
\end{aligned}
$$

(iii) $r(t)=r\left(t, \tau_{0}, z_{0}\right)$ is the maximal solution for

$$
z^{\prime}=G(t, z), z\left(\tau_{0}\right)=z_{0} \geq 0, \quad \text { for } t \geq \tau_{0}
$$

existing on $\left[\tau_{0}, \infty\right)$.

And let $\tilde{U}(t)=U\left(t-\eta, t_{0}, U_{0}\right), \tau_{0}=\eta+t_{0}$, where $U(t)$ $=\left(t, t_{0}, U_{0}\right)$ solves $(10)$ and $V(t)=V\left(t, \tau_{0}, V_{0}\right)$ solves (12) for $t \geq \tau_{0}$, and assume $L\left(\tau_{0}, V_{0}-U_{0}\right) \leq z_{0}$; then,

$$
L(t, V(t)-\tilde{U}(t)) \leq r(t), \quad \text { for } t \geq \tau_{0}
$$

Proof. Let

$$
m(t)=L(t, V(t)-\tilde{U}(t)), \quad \text { for } t \geq \tau_{0}
$$

For $t \geq \tau_{0}$, let us show the inequality $m(t) \leq r(t)$. As a matter of fact,

$$
\tilde{U}\left(\tau_{0}\right)=U\left(\tau_{0}-\eta, t_{0}, U_{0}\right), \tau_{0}=\eta+t_{0}
$$

Substituting the last statement in $m\left(\tau_{0}\right)$ together with the theorem's assumptions gives us

$$
\begin{aligned}
m\left(\tau_{0}\right) & =L\left(\tau_{0}, V\left(\tau_{0}\right)-\tilde{U}\left(\tau_{0}\right)\right) \\
& =L\left(\tau_{0}, V_{0}-U\left(\tau_{0}-\eta, t_{0}, U_{0}\right)\right) \\
& =L\left(\tau_{0}, V_{0}-U\left(t_{0}, t_{0}, U_{0}\right)\right) \\
& =L\left(\tau_{0}, V_{0}-U_{0}\right) \leq z_{0} .
\end{aligned}
$$

Let $\varepsilon>0$ be sufficiently small, and let us consider

$$
z^{\prime}=G(t, z)+\varepsilon, z\left(\tau_{0}\right)=z_{0}+\varepsilon, \quad \text { for } t \geq \tau_{0}
$$

whose we ensure the existence of its solutions $z(t, \varepsilon)=z(t$, $\left.\tau_{0}, z_{0}, \varepsilon\right)$ up to $r(t)$.

Let us conclude this proof by showing

$$
m(t)<z(t, \varepsilon), \quad \text { for } t \geq \tau_{0} .
$$

To do so, let us suppose on the contrary that there is a $t^{*} \geq \tau_{0}$ where

$$
m(t)<z(t, \varepsilon), \quad \text { for } t^{*}>t \geq \tau_{0}, m\left(t^{*}\right)=z\left(t^{*}, \varepsilon\right)
$$

Thus, it follows that

$$
D^{+} m\left(t^{*}\right) \geq m^{\prime}\left(t^{*}\right)=z^{\prime}\left(t^{*, \varepsilon}\right)=G\left(t^{*}, z\left(t^{*, \varepsilon}\right)\right)+\varepsilon .
$$

Taking into consideration the proposition regarding $G$ and since $G(t, z) \geq 0$, we conclude that the solutions $z(t, \varepsilon)$ are nondecreasing in $t$.

As $m(s)=L(s, V(s)-\tilde{U}(s))$ for $s \geq \tau_{0}$, we obtain

$$
L(s, V(s)-\tilde{U}(s)) \leq z\left(t^{*}, \varepsilon\right), \quad \text { for } t^{*} \geq s \geq \tau_{0}
$$

Consequently, $V-\tilde{U} \in E_{1}$. 
For sufficiently small enough $\delta>0$, we have

$$
\begin{aligned}
m(t+ & \delta)-m(t) \\
= & L(t+\delta, V(t+\delta)-\tilde{U}(t+\delta))-L(t, V(t)-\tilde{U}(t)) \\
\leq & L(t+\delta, V(t+\delta)-\tilde{U}(t+\delta))-L(t+\delta, V(t) \\
& -\tilde{U}(t)+\delta((P V)(t)-(\tilde{Q} \tilde{U})(t)))+L(t+\delta, V(t) \\
& -\tilde{U}(t)+\delta((P V)(t)-(\tilde{Q} \tilde{U})(t)))-L(t, V(t)-\tilde{U}(t)) \\
\leq & K D[[V(t+\delta)-V(t)]-[\tilde{U}(t+\delta)-\tilde{U}(t)] \\
& -\delta((P V)(t)-(\tilde{Q} \tilde{U})(t)), \tilde{0}]+L(t+\delta, V(t)-\tilde{U}(t) \\
& +\delta((P V)(t)-(\tilde{Q} \tilde{U})(t)))-L(t, V(t)-\tilde{U}(t)) .
\end{aligned}
$$

Hence, with considering the assumptions in (i) regarding the locally Lipschitzity of $L(t, W)$ in $W$, it is seen

$$
\begin{aligned}
m(t+\delta)-m(t) \leq & K\left[\varepsilon_{1}(\delta)-\varepsilon_{2}(\delta)\right]+L(t+\delta, V(t) \\
& -\tilde{U}(t)+\delta((P V)(t)-(\tilde{Q} \tilde{U})(t))) \\
& -L(t, V(t)-\tilde{U}(t)),
\end{aligned}
$$

where $\varepsilon_{1}$ and $\varepsilon_{2}$ stand for errors.

This gives us the following estimation regarding the Dini derivative of $m(t)$

$$
\begin{aligned}
D^{+} m(t) \leq & \lim _{\delta \longrightarrow 0^{+}} \sup \frac{1}{\delta} K\left[\varepsilon_{1}(\delta)-\varepsilon_{2}(\delta)\right] \\
& +\lim _{\delta \longrightarrow 0^{+}} \sup \frac{1}{\delta}[L(t+\delta, V(t)-\tilde{U}(t) \\
& +\delta((P V)(t)-(\widetilde{Q \tilde{U}})(t)))-L(t, V(t)-\tilde{U}(t))] \\
\leq & G(t, L(t, V(t)-\tilde{U}(t)))=G(t, m(t)),
\end{aligned}
$$

for $\tau_{0} \leq t \leq t^{*}<\infty$.

Since $\lim _{\delta \rightarrow 0}\left(\left(\varepsilon_{1}(\delta)-\varepsilon_{2}(\delta)\right) / \delta\right)=0$, when $t=t^{*}$, we have

$$
\begin{aligned}
D^{+} m\left(t^{*}\right) & \leq D_{*}^{+} L\left(t^{*}, V\left(t^{*}\right)-\tilde{U}\left(t^{*}\right)\right) \\
& \leq G\left(t^{*}, L\left(t, V\left(t^{*}\right)-\tilde{U}\left(t^{*}\right)\right)\right) \\
& =G\left(t^{*}, m\left(t^{*}\right)\right)<G\left(t^{*}, z\left(t^{*}, \varepsilon\right)\right)+\varepsilon
\end{aligned}
$$

which contradicts (55).
Consequently, we have $m(t)<z(t, \varepsilon)$, which in its turn, as $\varepsilon \longrightarrow 0$, gives us the desired estimation

$$
m(t) \leq r\left(t, \tau_{0}, z_{0}\right), \quad \text { for } t \geq \tau_{0} .
$$

Corollary 8. Assume that $L$ in Theorem 7 satisfies the aforementioned conditions in addition to that $G(t, z) \equiv 0$ and

$U \in E_{1}=\left\{\Omega \in K_{c}\left(\mathbb{R}^{n}\right): L(s, \Omega(s)) \leq L(t, \Omega(t)), \quad t_{0} \leq s \leq t\right\}$.

Then, $U(t)$ and $V(t)$ solve (10) and (11), respectively.

Equivalently, for $\tau_{0} \leq t^{*} \leq t^{* *}<\infty$, we have

$$
L\left(t^{* *}, V\left(t^{* *}\right)-U^{\sim}\left(t^{* *}\right)\right)<L\left(t^{*}, V\left(t^{*}\right)-\tilde{U}\left(t^{*}\right)\right) .
$$

\section{Theorem 9. Let}

(i) $L(t, W) \in C\left[\mathbb{R}_{+} \times K_{c}\left(\mathbb{R}^{n}\right), \mathbb{R}_{+}\right]$be locally Lipschitzian in $W$; i.e.,

$$
\left|L\left(t, W_{1}\right)-L\left(t, W_{2}\right)\right| \leq K D\left[W_{1}-W_{2}, \tilde{0}\right] .
$$

(ii) For $(t, V),(t, \tilde{U}) \in \mathbb{R}_{+} \times K_{c}\left(\mathbb{R}^{n}\right)$ and $G \in C\left[\mathbb{R}_{+} \times\right.$ $\left.\mathbb{R}_{+}, \mathbb{R}_{+}\right]$, we have

$$
\begin{aligned}
& A(t) D_{*}^{+} L(t, V(t)-\tilde{U}(t))+L(t, V(t)-\tilde{U}(t)) A^{\prime}(t) \\
& \quad \leq G(t, L(t, V(t)-\tilde{U}(t)) A(t)),
\end{aligned}
$$

for $t \geq \tau_{0}$ and $U \in E_{A}$ where

$$
\begin{aligned}
E_{A} & =\left\{\Omega \in K_{c}\left(\mathbb{R}^{n}\right): L(s, \Omega(s)) A(s)\right. \\
& \left.\leq L(t, \Omega(t)) A(t), \quad t_{0} \leq s \leq t\right\},
\end{aligned}
$$

and

$$
\begin{aligned}
D_{*}^{+} L(t, V(t)-\tilde{U}(t)) \\
=\lim _{\varepsilon \longrightarrow 0^{+}} \sup \frac{1}{\varepsilon}[L(t+\varepsilon, V(t)-\tilde{U}(t) \\
\quad+\varepsilon((P V)(t)-(\tilde{Q} \tilde{U})(t)))-L(t, V(t)-\tilde{U}(t))] .
\end{aligned}
$$

(iii) $r(t)=r\left(t, \tau_{0}, z_{0}\right)$ is the maximal solution of

$$
z^{\prime}=G(t, z), z\left(\tau_{0}\right)=z_{0} \geq 0, \quad \text { for } t \geq \tau_{0},
$$

existing on $\left[\tau_{0}, \infty\right)$.

And let $\tilde{U}(t)=U\left(t-\eta, t_{0}, U_{0}\right), \tau_{0}=\eta+t_{0}$, where $U(t)=$ $U\left(t, t_{0}, U_{0}\right)$ solves (10) and $V(t)=V\left(t, \tau_{0}, V_{0}\right)$ solves (12) 
for $t \geq \tau_{0}$, and assume

$$
A\left(\tau_{0}\right) L\left(\tau_{0}, V_{0}-U_{0}\right) \leq z_{0}
$$

Then,

$$
L(t, V(t)-\tilde{U}(t)) A(t) \leq r(t), \quad \text { for } t \geq \tau_{0} .
$$

Proof. Let

$$
M(t, V(t)-\tilde{U}(t))=L(t, V(t)-\tilde{U}(t)) A(t),
$$

for $t \geq \tau_{0}$, and assume $U(t) \in E_{A}$. Then, in view of the theorem's assumptions, we obtain

$$
\begin{aligned}
& D^{+} M(t, V(t)-\tilde{U}(t)) \\
& \leq A(t) D_{*}^{+} L(t, V(t)-\tilde{U}(t)) \\
&+L(t, V(t)-\tilde{U}(t)) A^{\prime}(t) \\
& \leq G(t, L(t, V(t)-\tilde{U}(t)) A(t)) \\
&= G(t, M(t, V(t)-\tilde{U}(t))) .
\end{aligned}
$$

The utilization of Theorem 7, in its turns, gives us the desired estimation

$$
L(t, V(t)-\tilde{U}(t)) A(t)=M(t, V(t)-\tilde{U}(t)) \leq r(t),
$$

for $t \geq \tau_{0}$.

After presenting the previous comparison theorems for ITD stability of SDE with causal operators, we can now employ them in proofing the following theorems regarding ITD stability, ITD asymptotic stability, and ITD uniformly asymptotic stability of the solution $U(t)=U\left(t, t_{0}, U_{0}\right)$ of the SDE (10).

\section{ITD Stability of SDEs with Causal Operators}

5.1. ITD Stability of SDEs with Causal Operators via Lyapunov Functions. Let us present sufficient conditions for stability of the solution $U(t)=U\left(t, t_{0}, U_{0}\right)$ of (10), using Lyapunov functions, and assuming the existence and uniqueness of this solution for $t \geq \tau_{0}$.

Theorem 10. Let

(i) $L(t, W) \in C\left[\mathbb{R}_{+} \times S(\rho), \mathbb{R}_{+}\right]$be be locally Lipschitzian in $W$; i.e.,

$$
\left|L\left(t, W_{1}\right)-L\left(t, W_{2}\right)\right| \leq K D\left[W_{1}-W_{2}, \tilde{0}\right] .
$$

(ii) For $(t, V),(t, \tilde{U}) \in \mathbb{R}_{+} \times S(\rho)$ and for $t \geq \tau_{0}$, and $U$ $\in E_{1}$

$$
D_{*}^{+} L(t, V-\tilde{U}) \leq 0,
$$

where

$$
\begin{aligned}
D_{*}^{+} L(t, V(t)-\tilde{U}(t)) \\
=\lim _{\varepsilon \longrightarrow 0^{+}} \sup \frac{1}{\varepsilon}[L(t+\varepsilon, V(t)-\tilde{U}(t) \\
\quad+\varepsilon((P V)(t)-(\tilde{Q} \tilde{U})(t)))-L(t, V(t)-\tilde{U}(t))] .
\end{aligned}
$$

(iii) $L(t, W)$ is positive definite and decrescent on $\mathbb{R}_{+} \times S$ $(\rho)$; i.e., there exists two functions $a, b$ from the class $\mathbb{K}$ such that

$a(D[W, \tilde{0}]) \leq L(t, W) \leq b(D[W, \tilde{0}]),(t, W) \in \mathbb{R}_{+} \times S(\rho)$

Then, the solution $V\left(t, \tau_{0}, V_{0}\right)$ of (12) is ITD stable w.r.t. the solution $\tilde{U}\left(t, \tau_{0}, U_{0}\right)=U\left(t-\eta, t_{0}, U_{0}\right)$ of (10), where $U\left(t, t_{0}, U_{0}\right)$ solves (10), for $t \geq \tau_{0}$.

Proof. Given a sufficiently small $\varepsilon>0$ and $t \geq \tau_{0}$, let us designate $\delta_{1}\left(\varepsilon, \tau_{0}\right)>0$ and $\delta_{2}\left(\varepsilon, \tau_{0}\right)>0$, with the aim that $b(\delta)<$ $a(\varepsilon)$ holds, where

$$
\delta\left(\varepsilon, \tau_{0}\right)=\max \left\{\delta_{1}\left(\varepsilon, \tau_{0}\right), \delta_{2}\left(\varepsilon, \tau_{0}\right)\right\}>0 .
$$

Then, with this $\delta$, we shall prove the stability of the solution $U\left(t, t_{0}, U_{0}\right)$ of (10) for $t \geq t_{0}$.

If we suppose the opposite, then there would exist solutions $V\left(t, \tau_{0}, V_{0}\right)$ of $(12)$ and $\tilde{U}\left(t, \tau_{0}, U_{0}\right)$ of $(10)$, on $\left[\tau_{0}, \infty\right.$ ) , and $t^{* *}>t^{*}>\tau_{0}$ so

$$
\begin{aligned}
D\left[V\left(t^{*}\right)-\tilde{U}\left(t^{*}\right), \tilde{0}\right] & =\delta, \\
D\left[V\left(t^{* *}\right)-\tilde{U}\left(t^{* *}\right), \tilde{0}\right] & =\varepsilon, \\
\delta \leq D[V(t)-\tilde{U}(t), \tilde{0}] & \leq \varepsilon, \quad \text { for } t^{*} \leq t \leq t^{* *} .
\end{aligned}
$$

By the assumption (ii) and Corollary 8 we obtain the following estimation

$$
\begin{aligned}
& L\left(t^{* *}, V\left(t^{* *}\right)-\tilde{U}\left(t^{* *}\right)\right) \\
& \quad \leq L\left(t^{*}, V\left(t^{*}\right)-\tilde{U}\left(t^{*}\right)\right), \quad \text { for } t^{*} \leq t \leq t^{* *}<\infty .
\end{aligned}
$$

Hence, employing the previous assumptions, especially (79), (80), and (iii), together with the choice of $\delta$, gives us that

$$
\begin{aligned}
a(\varepsilon) & =a\left(D\left[V\left(t^{* *}\right)-\tilde{U}\left(t^{* *}\right), \tilde{0}\right]\right) \\
& \leq L\left(t^{* *}, V\left(t^{* *}\right)-\tilde{U}\left(t^{* *}\right)\right) \\
& \leq L\left(t^{*}, V\left(t^{*}\right)-\tilde{U}\left(t^{*}\right)\right) \\
& \leq b\left(D\left[V\left(t^{*}\right)-\tilde{U}\left(t^{*}\right), \tilde{0}\right]\right)=b(\delta)<a(\varepsilon),
\end{aligned}
$$

which is a contradiction.

Therefore, we conclude the stability of the solution $U(t$, $\left.t_{0}, U_{0}\right)$ of (10) for $t \geq t_{0}$. Hence, the solution $V\left(t, \tau_{0}, V_{0}\right)$ of 
(12) is ITD stable w.r.t. the solution $\tilde{U}\left(t, \tau_{0}, U_{0}\right)=U(t-\eta$, $\left.t_{0}, U_{0}\right)$ of (10), assuming $U\left(t, t_{0}, U_{0}\right)$ solves (10), for $t \geq \tau_{0}$.

If additionally both $\delta_{1}=\delta_{1}\left(\varepsilon, \tau_{0}\right)$ and $\delta_{2}=\delta_{2}\left(\varepsilon, \tau_{0}\right)$ are independent of $\tau_{0}$, then the solution $U\left(t, t_{0}, U_{0}\right)$ of (10) is uniformly stable for $t \geq t_{0}$.

\section{Theorem 11. Let}

(i) $L(t, W) \in C\left[\mathbb{R}_{+}, S(\rho), \mathbb{R}_{+}\right]$be locally Lipschitzian in $W$; i.e.,

$$
\left|L\left(t, W_{1}\right)-L\left(t, W_{2}\right)\right| \leq K D\left[W_{1}-W_{2}, \tilde{0}\right]
$$

(ii) For $(t, V),(t, \tilde{U}) \in \mathbb{R}_{+} \times S(\rho)$ and for $t \geq \tau_{0}$, and $U$ $\in E_{A}$ where

$$
\begin{aligned}
E_{A} & =\left\{\Omega \in K_{c}\left(\mathbb{R}^{n}\right): L(s, \Omega(s)) A(s)\right. \\
& \left.\leq L(t, \Omega(t)) A(t), \quad t_{0} \leq s \leq t\right\}
\end{aligned}
$$

the following inequality holds

$$
D_{*}^{+} L(t, V(t)-\tilde{U}(t)) A(t)+L(t, V(t)-\tilde{U}(t)) A^{\prime}(t) \leq 0
$$

where

$$
\begin{aligned}
D_{*}^{+} L(t, V(t)-\tilde{U}(t)) \\
=\lim _{\varepsilon \longrightarrow 0^{+}} \sup \frac{1}{\varepsilon}[L(t+\varepsilon, V(t)-\tilde{U}(t) \\
\quad+\varepsilon((P V)(t)-(\tilde{Q} \tilde{U})(t)))-L(t, V(t)-\tilde{U}(t))] .
\end{aligned}
$$

Additionally, $A\left(\tau_{0}\right)=1, A^{\prime}(t)$ is continuous for $t \geq$ $\tau_{0}, A(t) \geq 1$, and $\lim _{t \rightarrow \infty} A(t)=\infty$, and

(iii) $L(t, W)$ is positive definite and decrescent on $\mathbb{R}_{+} \times S$ $(\rho)$; i.e., there exists two functions $a, b$ from the class $\mathbb{K}$ such that

$$
a(D[W, \tilde{0}]) \leq L(t, W) \leq b(D[W, \tilde{0}]),(t, W) \in \mathbb{R}_{+} \times S(\rho)
$$

Then, the solution $V\left(t, \tau_{0}, V_{0}\right)$ of (12) is ITD asymptotically stable w.r.t. the solution $\tilde{U}\left(t, \tau_{0}, U_{0}\right)=U\left(t-\eta, t_{0}, U_{0}\right)$ of (10) where $U\left(t, t_{0}, U_{0}\right)$ solves (10), for $t \geq \tau_{0}$.

Proof. Theorem 9 yields

$$
L(t, V(t)-\tilde{U}(t)) A(t) \leq L\left(\tau_{0}, V_{0}-U_{0}\right) \text { for } t \geq \tau_{0}
$$

Furthermore, by Theorem 10, we have the stability of the solution $U\left(t, t_{0}, U_{0}\right)$ of (10) for $t \geq t_{0}$. Therefore, it is suffi- cient to prove its quasiasymptotic stability property. To do this, let $\varepsilon=\rho$ so that

$$
\delta_{0}=\delta\left(\rho, \tau_{0}\right)=\max \left\{\delta_{1}\left(\rho, \tau_{0}\right), \delta_{2}\left(\rho, \tau_{0}\right)\right\}>0
$$

Choose $D\left[V_{0}-U_{0}, \tilde{0}\right]<\delta_{0}$ and $d_{s}\left[\tau_{0}-t_{0}, 0\right]<\delta_{2}$. Then, by using (ii), (iii), and (87), and since $\lim _{t \rightarrow \infty} A(t)=\infty$, i.e., $\lim _{t \rightarrow \infty} A^{-1}(t)=0$, it follows that given an $\varepsilon>0$ and $\tau_{0}$ $\geq 0$, a positive $T=T\left(\varepsilon, \tau_{0}\right)$ can be designated in order to satisfy

$$
\begin{aligned}
a(D[V(t)-\tilde{U}(t), \tilde{0}]) & \leq L(t, V(t)-\tilde{U}(t)) \\
& \leq L\left(\tau_{0}, V_{0}-U_{0}\right) A^{-1}(t) \\
& \leq b\left(D\left[V_{0}-U_{0}, \tilde{0}\right]\right) A^{-1}(t) \\
& <b\left(\delta_{0}\right) A^{-1}(t)<\varepsilon,
\end{aligned}
$$

given that $D\left[V_{0}-U_{0}, \tilde{0}\right]<\delta_{0}$ for $t \geq \tau_{0}+T$.

Therefore, the solution $U\left(t, t_{0}, U_{0}\right)$ of (10) is a quasiasymptotically stable solution for $t \geq t_{0}$. Hence, the solution $V\left(t, \tau_{0}, V_{0}\right)$ of (12) is ITD asymptotically stable w.r.t. the solution $\tilde{U}\left(t, \tau_{0}, U_{0}\right)=U\left(t-\eta, t_{0}, U_{0}\right)$ of (10), or the solution $U\left(t, t_{0}, U_{0}\right)$ of (10) is an asymptotically stable solution for $t \geq t_{0}$.

If additionally $\delta_{1}\left(\varepsilon, \tau_{0}\right), \delta_{2}\left(\varepsilon, \tau_{0}\right)$ and $T\left(\varepsilon, \tau_{0}\right)$ are all independent of $\tau_{0}$, then the solution $U\left(t, t_{0}, U_{0}\right)$ of (10) is uniformly asymptotically stable for $t \geq t_{0}$.

5.2. ITD Stability of SDEs with Causal Operators via Lyapunov Functionals. In the following, we present sufficient conditions for ITD uniformly asymptotic stability for SDEs with causal operators, in correspondence to Lyapunov second method, by using Lyapunov functional.

Theorem 12. Let

(i) $L(t, W) \in C\left[\mathbb{R}_{+} \times E, \mathbb{R}_{+}\right]$and satisfies the following estimation

$$
D^{+} L(t, V(t)-\tilde{U}(t)) \leq-c(D[V(t)-\tilde{U}(t), \tilde{0}]), \quad c \in \mathbb{K} .
$$

(ii) $L(t, W)$ is positive definite and decrescent on $\mathbb{R}_{+} \times E$; i.e., there exists two functions $a, b$ from the class $\mathbb{K}$ such that

$a(D[W, \tilde{0}]) \leq L(t, W) \leq b(D[W, \tilde{0}]),(t, W) \in \mathbb{R}_{+} \times E$

Then, the solution $V\left(t, \tau_{0}, V_{0}\right)$ of (12) is ITD uniformly asymptotically stable w.r.t. the solution $\tilde{U}\left(t, \tau_{0}, U_{0}\right)=U(t-$ $\left.\eta, t_{0}, U_{0}\right)$ of (10) where $U\left(t, t_{0}, U_{0}\right)$ solves (10), for $t \geq \tau_{0}$. 
Proof. Given a sufficiently small $\varepsilon>0$ and $t \geq \tau_{0}$, let us designate $\delta_{1}\left(\varepsilon, \tau_{0}\right)=\delta_{1}(\varepsilon)>0$ and $\delta_{2}\left(\varepsilon, \tau_{0}\right)=\delta_{2}(\varepsilon)>0$ so

$$
b(\delta)<a(\varepsilon)
$$

where $\delta(\varepsilon)=\max \left\{\delta_{1}(\varepsilon), \delta_{2}(\varepsilon)\right\}>0$.

Then, with this $\delta$ and $\varepsilon$, we shall prove the stability of the solution $U\left(t, t_{0}, U_{0}\right)$ of $(10)$ for $t \geq t_{0}$.

If we suppose the opposite, then there would exist solutions $V\left(t, \tau_{0}, V_{0}\right)$ of (12) and $\tilde{U}\left(t, \tau_{0}, U_{0}\right)$ of (10) for $t \geq \tau_{0}$, and $t_{1}>\tau_{0}$ so

$$
\begin{aligned}
& D\left[V\left(t_{1}, \tau_{0}, V_{0}\right)-\tilde{U}\left(t_{1}, \tau_{0}, U_{0}\right), \tilde{0}\right]=\varepsilon, \\
& D\left[V\left(t, \tau_{0}, V_{0}\right)-\tilde{U}\left(t, \tau_{0}, U_{0}\right), \tilde{0}\right] \leq \varepsilon, \quad \tau_{0} \leq t \leq t_{1} .
\end{aligned}
$$

Then, the assumption (ii) and Corollary 8 give us the following estimate

$$
L\left(t, V\left(t, \tau_{0}, V_{0}\right)-\tilde{U}\left(t, \tau_{0}, U_{0}\right)\right) \leq L\left(\tau_{0}, V_{0}-U_{0}\right)
$$

for $\tau_{0} \leq t \leq t_{1}<\infty$.

Hence, in view of (92), (93), (94), and (ii), in addition to the assumptions on $\delta$, we have the following statement

$$
\begin{aligned}
a(\varepsilon) & =a\left(D\left[V\left(t_{1}, \tau_{0}, V_{0}\right)-\tilde{U}\left(t_{1}, \tau_{0}, U_{0}\right), \tilde{0}\right]\right) \\
& \leq L\left(t_{1}, V\left(t_{1}, \tau_{0}, V_{0}\right)-\tilde{U}\left(t_{1}, \tau_{0}, U_{0}\right)\right) \\
& \leq L\left(\tau_{0}, V_{0}-U_{0}\right) \leq b\left(D\left[V_{0}-U_{0}, \tilde{0}\right]\right) \\
& =b(\delta)<a(\varepsilon),
\end{aligned}
$$

which is a contradiction. This yields that the solution $U(t$, $\left.t_{0}, U_{0}\right)$ of the unperturbed differential system (10) is uniformly stable for $t \geq t_{0}$.

To complete the proof, let us prove the uniformly asymptotic stability. Assume $\varepsilon=\rho$ and let $\delta_{0}=\delta(\rho)$ such that $D[$ $\left.V_{0}-U_{0}, \tilde{0}\right]<\delta_{0}, d_{s}\left[\tau_{0}-t_{0}, 0\right]<\delta_{0}$ gives us

$$
D\left[V\left(t, \tau_{0}, V_{0}\right)-U\left(t-\eta, t_{0}, U_{0}\right), \tilde{0}\right]<\rho \text { for } t \geq \tau_{0}
$$

Taking into consideration the uniform stability, it follows the existence of such $t^{*}$ that satisfies for $\tau_{0}<t^{*}<\tau_{0}+T$,

$$
\begin{aligned}
& D\left[V_{0}-U_{0}, \tilde{0}\right]<\delta_{0} \text { and } D\left[V\left(t^{*}, \tau_{0}, V_{0}\right)\right. \\
& \left.\quad-\tilde{U}\left(t^{*}, \tau_{0}, U_{0}\right), \tilde{0}\right]<\delta(\varepsilon),
\end{aligned}
$$

with $\delta=\delta(\varepsilon)>0$ corresponds to $\varepsilon>0$ for uniform stability.

If we assume that it is not true, let

$$
\delta \leq D\left[V\left(t, \tau_{0}, V_{0}\right)-\tilde{U}\left(t, \tau_{0}, U_{0}\right), \tilde{0}\right], t \in\left[\tau_{0}, \tau_{0}+T\right]
$$

Then, in view of (i), it leads to

$$
\begin{aligned}
& D^{+} L\left(t, V\left(t, \tau_{0}, V_{0}\right)-\tilde{U}\left(t, \tau_{0}, U_{0}\right)\right) \\
& \quad \leq-c\left(D\left[V\left(t, \tau_{0}, V_{0}\right)-\tilde{U}\left(t, \tau_{0}, U_{0}\right), \tilde{0}\right]\right)
\end{aligned}
$$

Hence, for $t \in\left[\tau_{0}, \tau_{0}+T\right]$, we obtain

$$
\begin{aligned}
& L\left(t, V\left(t, \tau_{0}, V_{0}\right)-\tilde{U}\left(t, \tau_{0}, U_{0}\right)\right) \\
& \quad \leq L\left(\tau_{0}, V_{0}-U_{0}\right)-\int_{\tau_{0}}^{t} c\left(V\left(s, \tau_{0}, V_{0}\right)-\tilde{U}\left(s, \tau_{0}, U_{0}\right)\right) d s .
\end{aligned}
$$

Since $b \in \mathbb{K}$, i.e., $b$ associates zero to zero and $b(\lambda)$ is strictly increasing in $\lambda$, and considering $D\left[V_{0}-U_{0}, \tilde{0}\right]<\delta_{0}$ we obtain that

$$
b\left(D\left[V_{0}-U_{0}, \tilde{0}\right]\right) \leq b\left(\delta_{0}\right)
$$

By substituting each $t$ by $\tau_{0}+T$ in the inequality (100) and by choosing

$$
T=1+\frac{b\left(\delta_{0}\right)}{c(\delta)},
$$

with the above assumptions, we have

$$
\begin{aligned}
0 & \leq L\left(\tau_{0}+T, V\left(\tau_{0}+T, \tau_{0}, V_{0}\right)-\tilde{U}\left(\tau_{0}+T, \tau_{0}, U_{0}\right)\right) \\
& \leq L\left(\tau_{0}, V_{0}-U_{0}\right)-\int_{\tau_{0}}^{\tau_{0}+T} c\left(V\left(s, \tau_{0}, V_{0}\right)-\tilde{U}\left(s, \tau_{0}, U_{0}\right)\right) d s \\
& \leq b\left(D\left[V_{0}-U_{0}, \tilde{0}\right]\right)-\int_{\tau_{0}}^{\tau_{0}+T} c\left(V\left(s, \tau_{0}, V_{0}\right)-\tilde{U}\left(s, \tau_{0}, U_{0}\right)\right) d s \\
& \leq b\left(\delta_{0}\right)-c(\delta) T=b\left(\delta_{0}\right)-c(\delta)\left(1+\frac{b\left(\delta_{0}\right)}{c(\delta)}\right) \\
& =b\left(\delta_{0}\right)-c(\delta)-b\left(\delta_{0}\right)=-c(\delta)<0,
\end{aligned}
$$

giving us a contradiction. Hence, there exists a $t^{*}>\tau_{0}$ such that

$$
D\left[V\left(t^{*}, \tau_{0}, V_{0}\right)-U\left(t^{*}-\eta, t_{0}, U_{0}\right), \tilde{0}\right]<\delta,
$$

which in its turn, by the stability property, gives us that

$$
D\left[V\left(t, \tau_{0}, V_{0}\right)-U\left(t-\eta, t_{0}, U_{0}\right), \tilde{0}\right]<\varepsilon,
$$

for $t \geq \tau_{0}+T$, given that

$$
D\left[V_{0}-U_{0}, \tilde{0}\right]<\delta \text { and } d_{s}\left[\tau_{0}-t_{0}, 0\right]<\delta
$$




\section{Conclusion}

Despite the obstacles we faced when trying to infer ITD stability properties from those ones concerning the classical notion of stability of the null solution, using a change-ofvariable approach, we managed to resolve those difficulties via comparison theorems for ITD stability of SDEs with causal operators, that take into consideration the change in initial conditions regarding time and position.

In this manuscript, Lyapunov functions and Lyapunov functionals are utilized to predict ITD stability, ITD asymptotic stability, and ITD uniformly asymptotic stability of the solutions of perturbed forms of SDEs involving causal operators corresponding to unperturbed systems, in light of the classical stability properties of the trivial solution of suitable comparison systems.

\section{Data Availability}

No data were used to support this study.

\section{Conflicts of Interest}

The authors declare that they have no conflicts of interest.

\section{Acknowledgments}

The authors would like to thank the Presidency for Turks Abroad and Related Communities (YTB) of Republic of Turkey Ministry of Culture and Tourism, and Gebze Technical University, for their support.

\section{References}

[1] V. Lakshmikantham, S. Leela, and A. Vatsala, "Setvalued hybrid differential equations and stability in terms of two measures," Hybrid Systems, vol. 2, no. 2, pp. 169-188, 2002.

[2] V. Lakshmikantham, T. G. Bhaskar, and J. V. Devi, Theory of Set Differential Equations in Metric Spaces, CSP, Cambridge Scientific Publ, Cottenham, Cambridge, 2006.

[3] Z. Drici, F. A. Mcrae, and J. Vasundhara Devi, "Stability results for set differential equations with causal maps," Dynamic Systems and Applications, vol. 15, no. 3, pp. 451-464, 2006.

[4] J. V. Devi and C. A. Naidu, "Stability results for set differential equations involving causal operators with memory," European Journal of Pure and Applied Mathematics, vol. 5, no. 2, pp. 187-196, 2012.

[5] V. Lakshmikantham and S. Leela, Differential and Integral Inequalities: Theory and Applications. 1: Ordinary Differential Equations, Academic Press, New York, 1969.

[6] V. Lakshmikantham, S. Leela, and A. A. Martyniuk, Stability Analysis of Nonlinear Systems, M. Dekker, New York, 1989.

[7] F. Brauer and J. A. Nohel, The Qualitative Theory of Ordinary Differential Equations: an Introduction, Dover ed, Dover, New York, N.Y, 1989.

[8] O. Kaleva, "The Cauchy problem for fuzzy differential equations," Fuzzy Sets and Systems, vol. 35, no. 3, pp. 389-396, 1990.

[9] C. Corduneanu, Functional Equations with Causal Operators. London, Taylor \& Francis, New York, 2002.
[10] V. Lakshmikantham, S. Leela, Z. Drici, and F. A. McRae, Theory of Causal Differential Equations, vol. 5, Atlantis Press, Paris, 2010.

[11] M. Arslan and C. Yakar, "Terminal value problems with causal operators," Hacettepe Journal of Mathematics and Statistics, vol. 48 , no. $5,2018$.

[12] V. Lakshmikantham, S. Leela, and J. Vasundhara Devi, "Stability theory for set differential equations," Dynamics of Continuous Discrete and Impulsive Systems Series A, vol. 11, no. Number 2-3, pp. 181-190, 2004.

[13] V. Lakshmikantham and M. R. M. Rao, Theory of IntegroDifferential Equations, Gordon and Breach Science Publishers, Lausanne, Switzerland, 1995.

[14] A. N. Chadaram, D. B. Dhaigude, and V. D. Jonnalagadda, "Stability results in terms of two measures for set differential equations involving causal operators," European Journal of Pure and Applied Mathematics, vol. 10, no. 4, pp. 645-654, 2017.

[15] C. Yakar and M. D. Shaw, "Initial time difference stability in terms of two measures and variational comparison result," Dynamics of Continuous, Discrete and Impulsive Systems, vol. 15, no. 3, pp. 417-425, 2008.

[16] C. Yakar and M. D. Shaw, "Practical stability in terms of two measures with initial time difference," Nonlinear Analysis: Theory Methods \& Applications, vol. 71, no. 12, pp. e781e785, 2009.

[17] C. Yakar and M. Çiçek, "Theory, methods and applications of initial time difference, boundedness and Lagrange stability in terms of two measures for nonlinear systems," Hacettepe Journal of Mathematics and Statistics, vol. 40, pp. 305330, 2011.

[18] C. Yakar, M. Çiçek, and M. B. Gücen, "Practical stability, boundedness criteria and Lagrange stability of fuzzy differential systems," Computers \& Mathematcs with Applications, vol. 64, no. 6, pp. 2118-2127, 2012.

[19] M. B. Gücen and C. Yakar, "Strict stability of fuzzy differential equations by Lyapunov functions," International Journal of Computer and Information Engineering, vol. 12, no. 5, pp. 315-319, 2018.

[20] T. G. Bhaskar and J. V. Devi, "Stability criteria for set differential equations," Mathematical and Computer Modelling, vol. 41, no. 11-12, pp. 1371-1378, 2005.

[21] T. G. Bhaskar and J. V. Devi, "Nonuniform stability and boundedness criteria for set differential equations," Applicable Analysis, vol. 84, no. 2, pp. 131-143, 2005.

[22] C. Yakar and M. D. Shaw, "A comparison result and Lyapunov stability criteria with initial time difference," Dynamics of Continuous, Discrete and Impulsive Systems, vol. 12, no. 6, pp. 731737, 2005.

[23] C. Yakar, "Boundedness criteria in terms of two measures with initial time difference. dynamics of continuous, discrete and impulsive systems. Series A: Mathematical analysis," in Proceedings of the 5th International Conference on Differential Equations and Dynamical Systems, pp. 270-275, Edinburg, Texas, USA, December 16-18, 2006.

[24] C. Yakar and M. Çiçek, "Initial time difference boundedness criteria and Lagrange stability," Dynamics of Continuous, Discrete and Impulsive Systems, vol. 18, no. Number 6 (7), pp. 797-811, 2011.

[25] C. Yakar, M. Çiçek, and M. B. Gücen, "Boundedness and Lagrange stability of fractional order perturbed system related 
to unperturbed systems with initial time difference in Caputo's Sense," Advances in difference Equations, vol. 2011, no. 1, 2011.

[26] M. Çiçek, C. Yakar, and B. Oğur, "Stability, boundedness, and Lagrange stability of fractional differential equations with initial time difference," Scientific World Journal, vol. 2014, pp. 1-7, 2014.

[27] C. Yakar and M. B. Gücen, "Initial time difference stability of causal differential systems in terms of Lyapunov functions and Lyapunov functionals," Journal of Applied Mathematics, vol. 2014, 7 pages, 2014. 